\title{
INFLUÊNCIA DO ARMAZENAMENTO NA QUALIDADE FISIOLÓGICA DE SEMENTES DE Caesalpinia pyramidalis Tul. ${ }^{1}$
}

\author{
Cimille Gabrielle Cardoso Antunes ${ }^{2}$, Claudineia Regina Pelacani ${ }^{3}$, Renata Conduru Ribeiro ${ }^{2}$, Hugo \\ Leonardo Rodrigues Gomes ${ }^{4}$ e Renato Delmondez de Castra ${ }^{4}$
}

\begin{abstract}
RESUMO - Objetivou-se com este trabalho apontar as melhores condições de armazenamento das sementes de C. pyramidalis (catingueira), visando ao estabelecimento de protocolos que possam servir de base para outras espécies do semiárido nordestino brasileiro. Sementes de catingueira foram armazenadas por 0, 3, 6 e 9 meses em ambiente de laboratório e em geladeira, nas embalagens saco de papel Kraft e saco de polietileno. A cada período de armazenamento, as sementes foram avaliadas quanto ao percentual de germinação (G\%), ao tempo médio de germinação $(\mathrm{Tm})$, à velocidade média de germinação $(\mathrm{Vm})$, ao índice de velocidade de germinação (IVG) e ao conteúdo de água (Ca). Observou-se elevada taxa de germinação das sementes nos diferentes tratamentos propostos aos três $(89,25 \%)$, seis $(88,5 \%)$ e nove (91\%) meses de armazenamento, sendo tais índices, inclusive, muito próximos ou ligeiramente superiores à taxa de germinação inicial (90\%). O armazenamento favoreceu significativamente a elevação das taxas de IVG das sementes para índices superiores àquele verificado nas sementes recém-coletadas (11,24 aos 3 meses; 9,79 aos 6 meses; 10,29 aos 9 meses e 7,70 no mês zero). Os resultados indicaram que as sementes de catingueira possuem comportamento ortodoxo quanto à tolerância à secagem e ao armazenamento e que a possibilidade de manutenção de sementes viáveis em diferentes ambientes a um baixo custo pode ser uma boa estratégia para fornecimento de sementes em qualquer época do ano.

Palavras-chave: Longevidade, Viabilidade e Germinação.
\end{abstract}

\section{THE STORAGE'S INFLUENCE IN THE PHYSIOLOGICAL QUALITY OF THE Caesalpinia pyramidalis TUL.}

\begin{abstract}
This work's objective was to point the best conditions for seed storage of C. pyramidalis (catingueira), aiming to establish protocols that can serve as a basis for other species of the Brazilian semi-arid northeast. Seeds of catingueira were stored for 0, 3, 6 and 9 months in laboratory environments and refrigerators in Kraft paper packaging bags and polyethylene bags. Every period of storage, seeds were assessed for Percentage of germination (G\%), the Average Time of germination (AT), the Average Speed of germination (AS), the Speed germination ( $S G)$ and Content of water $(C W)$. A high rate of seed germination in the different treatments that have been proposed was observed in the third (89.25\%), sixth (88.5\%) and ninth (91\%) months of storage, being such indexes even closest or slightly higher than those verified in the collected seeds (90\%). The storage favored significantly improved IVG seed rates, for higher indexes to those seen in collected seeds (11.24 third month; 9.79 sixth month; 10.29 ninth month and 7.70 collected seeds). The results suggest that catingueira seeds displayed an orthodox behavior as the tolerance for drying and storage, and the possibility of maintaining viable seeds in different environments at low cost can be a good strategy for the supply of seeds at any time of year.
\end{abstract}

Key-words: Longevity, Viability and Germination.

\footnotetext{
${ }^{1}$ Recebido em 06.11.2008 e aceito para publicação em 25.08.2010.

${ }^{2}$ Programa de Pós-Graduação em Engenharia Florestal pela Universidade Estadual de Feira de Santana, UEFS, Brasil.

${ }^{3}$ Universidade Estadual de Feira de Santana, UEFS, Brasil. E-mail: <pelacani@uefs.br>.

${ }^{4}$ Graduando em Engenharia Florestal pela Universidade Estadual de Feira de Santana, UEFS, Brasil.
} 


\section{INTRODUÇÃO}

A necessidade de conservação das florestas tropicais e o fortalecimento da política ambiental promoveram aumento da demanda de sementes de espécies nativas, que constituem insumo básico nos programas de recuperação e conservação de ecossistemas (CARVALHO et al., 2006). Contudo, ainda há carência de informações científicas que subsidiem tais programas, principalmente em áreas com maior abundância de grupos ecológicos (BARBEDO et al., 2002).

As estratégias para conservação da biodiversidade envolvem os métodos in situ e ex situ. A conservação in situ refere-se à manutenção das espécies no seu habitat por meio de unidades de conservação (como parques nacionais), enquanto o método de conservação ex situ consiste na conservação das espécies fora do seu habitat natural, de maneira complementar ao método in situ (FAO, 1993; BRASIL, 2000; BOTANIC GARDENS CONSERVATION INTERNATIONAL, 2001). A conservação ex situ pode ser realizada por meio do armazenamento de sementes, todavia o sucesso de tal procedimento depende do conhecimento sobre o comportamento destas quando armazenadas, o que possibilita a utilização de condições adequadas para a manutenção da viabilidade (HONG e ELLIS, 1996).

O armazenamento das sementes deve ser iniciado na maturidade fisiológica, e o maior desafio é conseguir que as sementes, após certo período, ainda apresentem elevada qualidade, prolongando a longevidade delas através do controle do grau de umidade, da temperatura e das condições do ambiente de armazenamento (CROCHEMORE, 1993; MEDEIROS, 2000). A decisão para a escolha das embalagens deve considerar, principalmente, as condições climáticas e as características mecânicas das embalagens (CARVALHO e NAKAGAWA, 2000; MARCOS FILHO, 2005; FERREIRA e BORGHETTI, 2004; BONOME, 2006).

A Caesalpinia pyramidalis Tul. (catingueira) é uma leguminosa endêmica da Caatinga com larga utilização no semiárido nordestino, devido ao seu potencial múltiplo, sendo sua madeira muito utilizada como carvão e estaca e suas folhas no tratamento de infecções diarreicas e catarrais (HARDESTY et al., 1988; NISHIZAWA et al., 1995; MAIA, 2004). O valor econômico da referida espécie vem desencadeando seu uso indiscriminado na Caatinga, visto que não há normatização institucional para o seu manejo
sustentável.Este trabalho objetivou determinar as melhores condições de armazenamento das sementes de catingueira, visando ao estabelecimento de protocolos que possam ser utilizados como modelo para outras espécies do Semiárido Nordestino brasileiro.

\section{MATERIAL E MÉTODOS}

As sementes foram obtidas a partir de 40 matrizes de catingueira localizadas em uma área aberta de caatinga, no Município de Petrolina, PE, sob as seguintes coordenadas geográficas: 9 30' 21'' S 40 30' 21'’ W. Segundo a classificação de Köppen, o clima nesta área apresenta-se como tropical semiárido, tipo BshW, seco e quente na parte norte e semiárido quente estípico na parte sul, caracterizado pela escassez e irregularidade das precipitações, com chuvas no verão e forte evaporação em consequência das altas temperaturas (AMBIENTE BRASIL, 2008).

C. pyramidalis é facilmente reconhecida quando ocorrem as primeiras manifestações de umidade, visto que suas gemas brotam rapidamente. Na estação chuvosa, tal espécie exibe porte arbóreo, chegando a atingir $10 \mathrm{~m}$ de altura, enquanto na estação seca ela se apresenta como arbusto tortuoso, com pouco mais de $2 \mathrm{~m}$ de altura. Quanto à fenologia da espécie, observou-se que, durante a estação seca, a catingueira adota comportamento de caducifolia, entretanto, 30 dias após o início das chuvas, ela alcança vegetação plena. A floração ocorre na época de transição-seca-chuvosa e na época chuvosa, seguida pela frutificação (MAIA, 2004).

Após a coleta, as sementes foram secas à sombra sob uma lona plástica em temperatura ambiente, durante três dias, com o intuito de reduzir o conteúdo de água delas, retardando possíveis degradações e alterações químicas dos tecidos seminais durante o armazenamento (NERY, 2006).

O beneficiamento e armazenamento das sementes foram realizados no Laboratório de Germinação de Sementes da Unidade Experimental Horto Florestal, da Universidade Estadual de Feira de Santana, em Feira de Santana, Bahia. O beneficiamento consistiu de avaliação visual das sementes, descartando-se aquelas danificadas, a fim de homogeneizar e purificar o lote. Após tal etapa, 3.200 sementes foram divididas em quatro grupos de 800 sementes (cada um deles correspondente a 0, 3, 6 e 9 meses de armazenamento), os quais foram subdivididos em quatro grupos de 200 sementes para 
cada embalagem testada (saco de papel Kraft permeável com dimensões de $15,0 \mathrm{~cm}$ x 10,7 cm x 0,08 mm e saco de polietileno semipermeável com dimensões de $38,8 \mathrm{~cm} \times 33,2 \mathrm{~cm} \times 0,06 \mathrm{~mm}$ ) no referido ambiente de armazenamento (geladeira a $8{ }^{\circ} \mathrm{C} \pm 2$ e temperatura ambiente de laboratório a $25^{\circ} \mathrm{C} \pm 3$ ). $\mathrm{O}$ armazenamento das sementes foi feito entre agosto de 2007 e maio de 2008.

A cada período de armazenamento, quatro repetições de 25 sementes de cada tratamento foram submetidas a testes de germinação com desinfestação prévia em hipoclorito de sódio 0,5\% durante 5', seguida de 1' em fungicida derosal $(1 \mathrm{~mL} / \mathrm{L})$. As sementes foram semeadas em caixas gerbox (esterilizadas em hipoclorito de sódio concentrado por 15') revestida por duas folhas de papel germitest embebidas em água destilada (sendo tal quantidade equivalente a 2,5 vezes o peso do substrato), posteriormente transferidas para câmaras de germinação (B.O.D.) ajustadas sob fotoperíodo de $12 \mathrm{~h}$ a $25^{\circ} \mathrm{C}$. As sementes foram avaliadas durante 13 dias, com troca do conjunto papel+gerbox a cada dois dias. Foram consideradas germinadas as sementes que apresentaram raiz primária com pelo menos $2 \mathrm{~mm}$ de comprimento, as quais foram descartadas após avaliação (BRASIL, 1992). As variáveis da germinação analisadas neste estudo foram: Porcentagem de germinação (G\%), Tempo médio de germinação (Tm), Velocidade média de germinação (Vm) e Índice de Velocidade de Germinação (IVG) (LABOURIAU, 1983).

Para acompanhamento do conteúdo de água das sementes durante o armazenamento, quatro repetições de 25 sementes foram submetidas à estufa de ventilação forçada a $60^{\circ} \mathrm{C}$ até atingir peso constante. O conteúdo de água foi obtido através da equação: (Pi-Pf)/Pi, em que $\mathrm{Pi}$ representa opeso inicial e o $\mathrm{Pf}$, o peso final das sementes (BRASIL, 1992).
O delineamento experimental proposto foi o inteiramente casualizado com quatro repetições de 25 sementes para cada tipo de armazenamento testado, em esquema fatorial $2 \times 2$. Os dados referentes à germinação e ao conteúdo de água das sementes foram expressos em porcentagem. Os índices de \% de germinação, tempo médio de germinação, velocidade média de germinação, índice de velocidade de germinação e conteúdo de água foram transformados segundo a função $(x+0,5)^{0,5}$. Após a normalização dos dados, estes foram submetidos à análise de variância e, em seguida, comparados pelo teste de Tukey a 5\% de probabilidade, através do programa estatístico SISVAR 4.3 (FERREIRA, 1999).

\section{RESULTADOS}

De acordo com os dados expressos na tabela de análise de variância (Tabela 1), verificou-se que a interação das fontes de variação (meses e tratamentos) foi somente significativa para o conteúdo de água das sementes, ao passo que para as demais variáveis da germinação as variáveis atuaram de maneira independente.

No que diz respeito ao conteúdo de água (Tabela 2), observou-se que, ao longo dos meses de armazenamento, somente a condição ambiente saco de papel Kraft propiciou aumento significativo no conteúdo de água das sementes (de 6,22\% no primeiro mês para 8,5\% no nono mês). Na condição ambiente saco de polietileno, as sementes apresentaram aumento no conteúdo de água no nono mês de armazenamento (7,23\%), mas sem diferenças significativas com relação aos meses três $(6,37 \%)$ e seis $(6,51 \%)$. Nas condições geladeira saco de papel Kraft e geladeira saco de polietileno, entretanto, houve redução no conteúdo de água das sementes, porém sem diferenças significativas entre os meses avaliados. Aparentemente houve efeito diferencial entre ambiente versus geladeira.

Tabela 1 - Análise de variância das variáveis cinéticas da germinação de sementes de Caesalpinia pyramidalis Tul. submetidas a diferentes tratamentos de deficiência hídrica e armazenadas por nove meses. Feira de Santana, Bahia, agosto de 2007 a fevereiro de 2008.

Table 1 - Analysis of variance of the Kinetic variables of the germination of Caesalpinia pyramidalis Tul. seeds subjected to differents treatments of water deficiency and stored for nine months. Feira de Santana, Bahia, August 2007 to February 2008.

\begin{tabular}{|c|c|c|c|c|c|}
\hline F. variação & G\% & Tm (dias) & Vm $\left(\right.$ dias $\left.^{-1}\right)$ & VG & CA \\
\hline Meses & $0,6771^{\text {ns }}$ & $0,0163 *$ & $0,1071^{\mathrm{ns}}$ & $0,0007^{*}$ & $0,0059 *$ \\
\hline Tratamentos & $0,0267 *$ & $0,0918^{\mathrm{ns}}$ & $0,0951^{\mathrm{ns}}$ & $0,0027 *$ & $0,0000 *$ \\
\hline $\mathrm{M} \times \mathrm{T}$ & $0,8314^{\mathrm{ns}}$ & $0,9328^{\mathrm{ns}}$ & $0,9917^{\mathrm{ns}}$ & $1,0000^{\mathrm{ns}}$ & $0,0002 *$ \\
\hline $\mathrm{CV} \%$ & 3,31 & 7,14 & 5,88 & 7,72 & 4,47 \\
\hline
\end{tabular}


A germinabilidade (Tabela 3) foi mantida em taxas elevadas aos três $(89,25 \%)$, seis $(88,5 \%)$ e nove (91\%) meses de armazenamento, sendo tais índices, inclusive, muito próximos, ou ligeiramente superiores à taxa de germinação inicial (90\%), independentemente da embalagem ou do tipo de ambiente utilizado para o acondicionamento.

Tabela 2 - Conteúdo de água das sementes de Caesalpinia pyramidalis Tul. armazenadas por seis meses, em diferentes embalagens e ambientes. Feira de Santana, Bahia, agosto de 2007 a maio de 2008. RC - Recém coletadas; ASP - Ambiente Saco de Papel; ASPL - Ambiente Saco de Polietileno; GSP - Geladeira Saco de Papel; e GSPL - Geladeira Saco de Polietileno.

Table 2 - Water content of Caesalpinia pyramidalis Tul. seeds stored for six months in different packages and environments. Feira de Santana, Bahia, August 2007 to May 2008. RC - Recently Collected; EPPB - Environment Paper Bag; EPB - Environment Polyethylene Bag; RPPB - Refrigerator Paper Bag; RPB - Refrigerator Polyethylene Bag.

\begin{tabular}{cccccr}
\hline \multirow{2}{*}{ Tratamentos } & \multicolumn{5}{c}{ Conteúdo de água (\%) } \\
\cline { 2 - 5 } & $\mathbf{0}$ & $\mathbf{3}$ & $\mathbf{6}$ & $\mathbf{9}$ \\
\hline RC & $6,22 \mathrm{Aa}$ & $/$ & $/$ & $/$ & Média \\
ASP & $/$ & $6,03 \mathrm{bA}$ & $6,02 \mathrm{bA}$ & $8,50 \mathrm{aA}$ & 6,22 \\
ASPL & $/$ & $6,51 \mathrm{aA}$ & $6,37 \mathrm{aA}$ & $7,23 \mathrm{aB}$ & 6,85 \\
GSP & $/$ & $4,83 \mathrm{aB}$ & $4,79 \mathrm{aB}$ & $4,58 \mathrm{aD}$ & 6,71 \\
GSPL & $/$ & $5,96 \mathrm{aA}$ & $5,68 \mathrm{aAB}$ & $5,81 \mathrm{aC}$ & 4,73 \\
Média & 6,22 & 5,83 & 5,71 & 6,53 & 5,82 \\
\hline
\end{tabular}

Médias seguidas pela mesma letra minúscula na linha e maiúscula na coluna não diferem entre si, pelo Teste de Tukey a $5 \%$ de probabilidade.

Medium followed by the same letter in the row and capitalizes column do not differ by Tukey Test test at 5\% probability.

Tabela 3 - Germinação e tempo médio de germinação de sementes de Caesalpinia pyramidalis Tul. armazenadas por nove meses, em diferentes embalagens e ambientes. Feira de Santana, Bahia, agosto de 2007 a maio de 2008. RC Recém Coletadas; ASP - Ambiente Saco de Papel; ASPL - Ambiente Saco de Polietileno; GSP - Geladeira Saco de Papel; GSPL - Geladeira saco de polietileno.

Table 3 - Germination and average time of germination of Caesalpinia pyramidalis Tul. seeds stored for six months in different packages and environments. Feira de Santana, Bahia, August 2007 to May 2008. RC - - Recently Collected; EPPB - - Environment Paper Bag; EPB - Environment Polyethylene Bag; RPPB - Refrigerator Paper Bag; RPB - Refrigerator Polyethylene Bag.

\begin{tabular}{|c|c|c|c|c|c|}
\hline \multicolumn{6}{|c|}{ Meses } \\
\hline \multirow[t]{2}{*}{ Tratamentos } & \multicolumn{4}{|c|}{ Germinação (\%) } & \multirow[t]{2}{*}{ Média } \\
\hline & $\mathbf{0}$ & 3 & 6 & 9 & \\
\hline RC & 90,00 & I & I & I & $90,00 \mathrm{~A}$ \\
\hline ASP & I & 87,00 & 83,00 & 84,00 & $84,67 \mathrm{~A}$ \\
\hline ASPL & / & 88,00 & 89,00 & 93,00 & $90,00 \mathrm{~A}$ \\
\hline GSP & / & 91,00 & 92,00 & 93,00 & $92,00 \mathrm{~A}$ \\
\hline GSPL & I & 91,00 & 90,00 & 94,00 & $91,67 \mathrm{~A}$ \\
\hline Média & $90,00 \mathrm{a}$ & $89,25 a$ & $88,50 a$ & $91,00 a$ & \\
\hline \multicolumn{6}{|c|}{ Tempo médio (dias) } \\
\hline RC & 2,50 & I & I & I & $2,50 \mathrm{~A}$ \\
\hline ASP & I & 1,79 & 2,24 & 2,13 & $2,05 \mathrm{~A}$ \\
\hline ASPL & I & 2,05 & 2,26 & 2,25 & $2,18 \mathrm{~A}$ \\
\hline GSP & I & 2,25 & 2,55 & 2,34 & $2,38 \mathrm{~A}$ \\
\hline GSPL & I & 1,92 & 2,63 & 2,58 & $2,38 \mathrm{~A}$ \\
\hline Média & $2,50 \mathrm{a}$ & $2,00 \mathrm{~b}$ & $2,42 \mathrm{ab}$ & $2,32 \mathrm{ab}$ & \\
\hline
\end{tabular}

Médias seguidas pela mesma letra minúscula na linha e maiúscula na coluna não diferem entre si, pelo Teste teste de Tukey a $5 \%$ de probabilidade.

Medium followed by the same letter in the row and capitalized column do not differ by Tukey test at $5 \%$ probability.

Revista Árvore, Viçosa-MG, v.34, n.6, p.1001-1008, 2010 
Com relação ao tempo médio de germinação (Tm), observou-se que as sementes de catingueira germinaram, em média, dentro de dois dias, mantendo tal característica em todas as condições de armazenamento estabelecidas. O Tm das sementes variou significativamente ao longo do período experimental, e as sementes com três meses de armazenamento requereram menor tempo para germinar (Tabela 3).

A velocidade média de germinação ( Vm) das sementes de catingueira não diferiu entre os tratamentos propostos e nem entre os meses estudados, muito embora aos três meses de armazenamento tenham sido observados os melhores índices, assim como para o tempo médio de germinação (Tabela 4).

Quanto ao índice de velocidade de germinação ou IVG, verificou-se que todos os tratamentos utilizados estimularam o aumento do vigor das sementes quando comparado com àquelas recémcoletadas (Tabela 4).

\section{DISCUSSÃO}

O conteúdo de água das sementes é um dos fatores que mais interferem na manutenção da pura qualidade fisiológica; assim, quanto mais baixo, maior a longevidade das sementes. Condições que possibilitem flutuações ambientais de temperatura e de umidade relativa propiciam variações no conteúdo de água das sementes, principalmente pelo potencial higroscópico que estas exibem (POPINIGIS, 1977). Assim, o comportamento das sementes de catingueira na condição ambiente saco de papel Kraft pode ser justificado pelo fato de esta não ser condição controlada, sendo o efeito da temperatura preponderante ao tipo de acondicionamento.

Geralmente, condições de baixa temperatura permitem a manutenção do conteúdo de água das sementes em níveis baixos e o metabolismo reduzido, e ambientes com temperaturas mais elevadas influenciam na perda mais rápida da viabilidade das sementes, por acelerar as reações metabólicas seminais e propiciar, muitas vezes, o aumento do conteúdo de água (FERREIRA e

Tabela 4 - Velocidade média de germinação e índice de velocidade de germinação de sementes de Caesalpinia pyramidalis Tul. armazenadas por nove meses, em diferentes embalagens e ambientes. Feira de Santana, Bahia, agosto de 2007 a maio de 2008. RC - Recém- Ccoletadas; ASP - Ambiente Saco de Papel; ASPL - Ambiente Saco de Polietileno; GSP - Geladeira Saco de Papel; e GSPL - Geladeira saco de polietileno.

Table 4 - Average speed of germination and Speed of germination of Caesalpinia pyramidalis Tul. seeds stored for six months in different packages and environments. Feira de Santana, Bahia, August 2007 to May 2008. RC - Recently Collected; EPPB - Environment Paper Bag; EPB - Environment Polyethylene Bag; RPPB - Refrigerator Paper Bag; RPB - Refrigerator Polyethylene Bag.

\begin{tabular}{|c|c|c|c|c|c|}
\hline \multicolumn{6}{|c|}{ Meses } \\
\hline \multirow[t]{2}{*}{ Tratamentos } & \multicolumn{4}{|c|}{ Velocidade média de germinação $\left(\right.$ dias $\left.^{-1}\right)$} & \multirow[t]{2}{*}{ Média } \\
\hline & $\mathbf{0}$ & 3 & 6 & 9 & \\
\hline RC & 0,40 & I & / & / & $0,40 \mathrm{~A}$ \\
\hline ASP & I & 0,62 & 0,52 & 0,49 & $0,55 \mathrm{~A}$ \\
\hline ASPL & / & 0,49 & 0,45 & 0,45 & $0,46 \mathrm{~A}$ \\
\hline GSP & / & 0,45 & 0,39 & 0,43 & $0,43 \mathrm{~A}$ \\
\hline GSPL & / & 0,52 & 0,39 & 0,39 & $0,43 \mathrm{~A}$ \\
\hline Média & $0,40 a$ & $0,52 \mathrm{a}$ & $0,44 a$ & $0,44 a$ & \\
\hline \multicolumn{6}{|c|}{ Índice de velocidade de germinação (IVG) } \\
\hline RC & 7,70 & l & / & l & $7,70 \mathrm{~B}$ \\
\hline ASP & / & 10,10 & 9,20 & 9,33 & $9,55 \mathrm{~A}$ \\
\hline ASPL & / & 11,30 & 10,39 & 10,72 & $10,80 \mathrm{~A}$ \\
\hline GSP & / & 11,16 & 9,99 & 11,04 & $10,73 \mathrm{~A}$ \\
\hline GSPL & / & 12,42 & 9,59 & 10,10 & $10,70 \mathrm{~A}$ \\
\hline Média & $7,70 b$ & $11,24 \mathrm{a}$ & 9,79a & $10,29 a$ & \\
\hline
\end{tabular}

Médias seguidas pela mesma letra minúscula na linha e maiúscula na coluna não diferem entre si, pelo Teste teste de Tukey a $5 \%$ de probabilidade.

Medium followed by the same letter in the row and capitalized column do not differ by Tukey test at 5\% probability. 
BORGHETTI, 2004). As sementes de catingueira apresentaram o referido perfil, enquanto Pontes et al. (2006) observaram efeito inverso com as sementes de Caesalpinia peltophoroides (Benth.), havendo aumento no teor de água das sementes em condições de refrigeração e redução na condição de temperatura mais elevada.

De acordo com Fowler (2000), baixos níveis de umidade reduzem as taxas respiratórias das sementes, resultando em maior vigor e viabilidade e prolongando, assim, a longevidade das sementes. Isso justifica, dessa forma, o elevado desempenho germinativo das sementes nas diferentes condições de armazenamento. Além disso, a qualidade inicial das sementes pode ter sido outro fator primordial para manutenção da germinabilidade. Perez et al. (1999), avaliando sementes de canafístula (Peltophorum dubim (Spreng) Taubert), também observaram que a viabilidade e o vigor não foram alterados após o armazenamento em ambiente natural ou a $10^{\circ} \mathrm{C}$ e com o uso de embalagem de papel ou vidro com tampa plástica após 45 e 70 dias de armazenamento. Pinho et al. (2009), estudando a germinação de sementes de Anadenanthera peregrina (L.) SPEG. durante 300 dias de armazenamento, também verificaram manutenção da germinabilidade a 5 e $20^{\circ} \mathrm{C}$. Araújo Neto et al. (2005), entretanto, avaliando a germinabilidade de sementes de monjoleiro submetidas a 24 meses de armazenamento, verificou diferenças na germinabilidade já partir do quarto mês experimental, com redução de tal variável nas sementes mantidas em ambiente de laboratório e conservação da capacidade germinativa das sementes acondicionadas em câmara fria. Barbedo et al. (2002) também encontraram diferenças na germinação de sementes de pau-brasil (Caesalpinia echinata Lam.), a depender da condição de armazenamento, sendo o ambiente de laboratório considerado negativo para o armazenamento das sementes e as condições de baixa temperatura, as mais recomendadas.

Com relação ao tempo médio de germinação (Tm), uma das variáveis que ajudam a expressar o vigor do lote, dando ao processo germinativo um caráter cinético (FERREIRA e BORGHETTI, 2004), o comportamento observado sugere que a espécie provavelmente adota em condições naturais, estratégias de estabelecimento rápido, para aproveitamento de condições ambientais favoráveis, característica típica de espécies pioneiras como C. pyramidalis (FERREIRA e BORGHETTI, 2004; MELO et al., 2004).
Os dados de velocidade média de germinação das sementes nos diferentes tratamentos estabelecidos concordam com aqueles referentes ao Tm, corroborando o fato de que tais grandezas são inversamente proporcionais, já que, quanto menor o tempo médio de germinação das sementes, maior a velocidade média de germinação destas, embora sem diferenças significativas entre os meses. Perez et al. (1999), no entanto, verificaram variações na velocidade média de germinação das sementes de canafístula, dependendo da condição de armazenamento empregada. A condição ambiente de laboratório promoveu redução da Vm, enquanto o acondicionamento em baixa temperatura promoveu a manutenção da Vm em índices próximos aos iniciais aos 150 dias de armazenamento.

Quanto ao índice de velocidade de germinação ou IVG, índice que permite inferir com precisão o vigor de um lote, haja vista que ele contabiliza a quantidade de sementes germinadas por unidade de tempo (MAGUIRE, 1962; NAKAGAWA, 1999; FERREIRA e BORGHETTI, 2004), pode-se destacar que todas as condições experimentais (independente da embalagem ou do tipo de acondicionamento) estimularam o aumento dessa variável, retardando possíveis alterações fisiológicas e bioquímicas típicas do processo de deterioração, que comprometem não só o vigor, como também a sobrevivência da semente durante o armazenamento (SANTOS et al., 2007). Araújo Neto et al. (2005), no entanto, observaram diferenças no vigor de sementes de monjoleiro em função do tipo de armazenamento aplicado. A condição embalagem de vidro em câmara fria foi a mais adequada para manutenção do vigor das sementes por dois anos, e a condição saco de papel em ambiente de laboratório foi a menos indicada para tanto, já que as sementes apresentaram baixas taxas de IVG a partir do oitavo mês de armazenamento.

\section{CONCLUSÃO}

A partir dos dados observados, conclui-se que a manutenção do conteúdo de água das sementes em níveis baixos, durante todos os meses de avaliação, foi o fator preponderante para a conservação do potencial germinativo das sementes (viabilidade), sugerindo que elas exibem comportamento ortodoxo quanto à tolerância à secagem e ao armazenamento. 
Os resultados indicam ainda que as sementes de catingueira podem ser armazenadas por períodos inferiores a um ano, tanto em temperatura ambiente de laboratório quanto em geladeira, utilizando-se para o acondicionamento embalagens permeáveis (sacos de papel Kraft) ou semipermeáveis (sacos de polietileno), desde que o conteúdo de água inicial das sementes seja baixo.

Além disso, a possibilidade de manutenção de sementes viáveis em diferentes ambientes a um baixo custo pode ser uma boa estratégia para fornecimento de sementes a qualquer época do ano.

\section{AGRADECIMENTOS}

Ao CNPq e à Embrapa/Semiárido, Petrolina, PE, especialmente na pessoa da Professora Dra. Bárbara Aragão, pelo fornecimento de material vegetal.

\section{REFERÊNCIAS}

AMBIENTE BRASIL. Classificação climática de Köppen-Geiger. 2008. Disponível em: <www.ambientebrasil.com.br> . Acesso em: 30/06/08.

ARAÚJO NETO, J. C. et al. Armazenamento e requerimento fotoblástico de sementes de Acacia polyphylla DC. Revista Brasileira de Sementes, v.27, n.1, p.115-124, 2005.

BARBEDO, C. J.; BILIA, D. A. C.; FIGUEIREDORIBEIRO, R. C. Tolerância à dessecação e armazenamento de sementes de Caesalpinia echinata Lam. (pau-brasil), espécie da Mata Atlântica. Revista Brasileira de Botânica, v.25, n.4, p.431-439, 2002.

BONOME, L. T. S. Alterações fisiológicas, bioquímicas e moleculares em sementes de seringueira [Hevea brasiliensis (willd. ex adr. de juss.) müell.-arg.] durante o armazenamento. 2006. 136f. Tese (Doutorado em Fisiologia Vegetal) - Universidade Federal de Lavras, Lavras, 2006.

\section{BOTANIC GARDENS CONSERVATION}

INTERNATIONAL. Normas internacionais de conservação para jardins botânicos. Rio de Janeiro: Conselho Nacional do Meio Ambiente/Rede Brasileira de Jardins Botânicos/ Instituto de Pesquisas e Jardim Botânico do Rio de Janeiro/EMC, 2001.
BRASIL. Ministério da Agricultura e Reforma Agrária. Regras para análise de sementes. Brasília: 1992. 365p.

BRASIL. Ministério da Agricultura e Reforma Agrária Convenção sobre diversidade biológica: conferência para adoção do texto acordado da CDB - Ato final de Nairobi. Brasília: 2000. 60p. (Biodiversidade, 2).

CARVALHO, N. M.; NAKAGAWA, J. Sementes: ciência, tecnologia e produção. 4.ed. Jaboticabal: Funep, 2000. 588p.

CARVALHO, L. R.; SILVA, E.A. A.; DAVIDE, A. C. Classificação de sementes florestais quanto ao comportamento no armazenamento. Revista Brasileira de Sementes, v.28, n.2, p.15-25, 2006.

CROCHEMORE, M. L. Conservação de sementes de tremoço azul (Lupunis angustifolius L.) em diferentes embalagens. Revista Brasileira de Sementes, v.15, n.2, p.227-231, 1993.

FAO. Ex situ sorage of seeds, pollen and in vitro cultures of perennial woody plant species. Rome: 1993. 83p. (Foresty Paper)

FERREIRA, D. F. SISVAR 4. 3 - Sistema de análises estatísticas. Lavras: Universidade Federal de Lavras, 1999.

FERREIRA, A. G.; BORGHETTI, F.

Germinação: do básico ao aplicado. Porto Alegre: Artmed, 2004. 323p.

FOWLER, J. A. P. Superação de dormência e armazenamento de sementes de espécies florestais. In: GALVÃO, A. P. M.

Reflorestamento de propriedades rurais para fins produtivos e ambientais: um guia para ações municipais e regionais. Brasília: Embrapa Comunicação para Transferência de Tecnologias, 2000. p.77-99.

HARDESTY, L. H.; BOX, T. W.; MALECHEK, J. C. Season of cutting affects biomass production by coppicing browse species of the Brazilian Caatinga. Journal of Range Management, v.41, n.6, p.447-80, 1988.

HONG, T. D.; LININGTON, S.; ELLIS, R. H. Seed storage behaviour: a compendium. Rome: IPGRI, 1996.

Revista Árvore, Viçosa-MG, v.34, n.6, p.1001-1008, 2010 
LABOURiAu, L. G. A germinação das sementes. Washington: Organização dos Estados Americanos, 1983. 170p. (Monografia Científica).

MAGUIRE, J. D. Speed of germination-aid in selection and evaluation for seedling emergence and vigor. Crop Science, v.1, p.176-177, 1962.

MAIA, G. N. Caatinga: árvores, arbustos e suas utilidades. São Paulo: D\&Z Computação Gráfica e Editora, 2004. 413p.

MARCOS FILHO, J. Fisiologia de sementes de plantas cultivadas. Piracicaba: FEALQ, 2005. 495p.

MEDEIROS, A. C. S. Armazenamento de sementes de espécies florestais de Mata Atlântica. In: VIBRANS, A. C.; GAlVÃO, P. Curso de manejo e conservação de sementes de especies arboreas da mata atlantica - Região sul, 1. Blumenau: URB/FURB/Embrapa, 2000. p.48-59.

MELO, F. P. L. et al. Recrutamento e estabelecimento de plântulas. In: FERREIRA, A. G.; BORGHETTI, F. Germinação: do básico ao aplicado. São Paulo: Artmed, 2004. p.237-250.

NAKAGAWA, J. Testes de vigor baseados no desempenho das plântulas. In: KRYZANOWSKI, F. C.; VIEIRA, R. D.; FRANÇA NETO, J. B. Vigor de sementes: conceitos e testes. Londrina: Abrates, 1999. p.2.1-2.24.

NERY, F. C. Aspectos da germinação, armazenamento de sementes, crescimento inicial e anatomia de plantas jovens de Calophyllum brasiliense Cambess. 2006. 173f. Dissertação (Mestrado em Fisiologia Vegetal) Universidade Federal de Lavras, Lavras, 2006.
NISHIZAWA, T.; TSUCHIYA, A.; PINTO, M. M. V. Characteristics and utilization of tree species in the semi-arid woodland of northeast Brazil. In: NISHIZAWA, T. \& UITTO, J. I. The fragile tropics of Latin America: sustainable management of changing environments. 1995. Disponível em: <http://www.unu.edu/unupress/ unupbooks/80877e/80877E00.htm>. Acesso em: 20/05/2008.

PEREZ, S. C. J. G. A.; FANTI, S. C.; CASALI, C. A. Influência do armazenamento, substrato, envelhecimento precoce e profundidade de semeadura na germinação de canafístula. Bragantia, v.58, n.1, p.57-68, 1999.

PINHO, D. S. et al. Avaliação da qualidade fisiológica de sementes de Anadenanthera peregrina (L.) SPEG. durante o armazenamento. Revista Árvore, v.33, n.1, p.27-33, 2009.

PONTES, C. A. et al. Influência da temperatura de armazenamento na qualidade das sementes de Caesalpinia peltophoroides Benth. (Sibipiruna). Revista Árvore, v.30, n.1, p.43-48, 2006.

POPINIGIS, F. Qualidade fisiológica de sementes. Sementes, v.1, n.1, p.65-80, 1977.

SANTOS, S. R. G.; PAULA, R. C. Qualidade fisiológica de sementes de Sebastiania commersoniana (Baill.) Smith \& Downs (branquilho - Euphorbiaceae) durante o armazenamento. Scientia Forestalis, v.74, p.87-94, 2007. 